\title{
A parsimonious diffusion equation for electricity demand
}

\author{
Elliot Tonkes ${ }^{1} \quad$ Phil Broadbridge ${ }^{2}$
}

(Received 15 February 2013; revised 2 January 2014)

\begin{abstract}
We present a parsimonious model for describing the stochastic dynamics of electricity demand in the NSW region of the National Electricity Market. We apply a moment matching approach to calibrate the parameters and perform in-sample and out-of-sample tests to demonstrate the model's capability and weaknesses. We show a solid improvement when the calibration uses the minimum and maximum daily temperatures in the regression. We clearly express the relationship between the drift term and the expected demand, which is a nontrivial connection and has not been made explicit in other publications.

Subject class: $60 \mathrm{G} 07$

Keywords: Electricity demand
\end{abstract}

http://journal . austms.org.au/ojs/index.php/ANZIAMJ/article/view/6751 gives this article, (C) Austral. Mathematical Soc. 2014. Published January 23, 2014, as part of the Proceedings of the 16th Biennial Computational Techniques and Applications Conference. ISSN 1446-8735. (Print two pages per sheet of paper.) Copies of this article must not be made otherwise available on the internet; instead link directly to this URL for this article. 


\section{Contents}

1 Introduction

C748

2 Electricity demand

C749

3 Model formulation

C750

3.1 Stochastic differential equation . . . . . . . . . . . C750

3.2 Characteristics of SDE solution . . . . . . . . . . C751

3.3 Demand regression model . . . . . . . . . . . C753

4 Model calibration approach

C754

4.1 Literature . . . . . . . . . . . . . . . . . C754

4.2 Methodology for calibration of the SDE . . . . . . . C755

5 Case study: NSW region of the NEM

C757

5.1 Data sources . . . . . . . . . . . . . . . . C757

5.2 Regression fit . . . . . . . . . . . . C C757

5.3 Calibration of the SDE . . . . . . . . . . C758

5.4 Results . . . . . . . . . . . . . . . . . C759

5.4 .1 In sample simulations . . . . . . . . . . . C C760

5.4 .2 Out of sample simulations . . . . . . . . . C C762

5.4 .3 Earth Hour . . . . . . . . . . . . . . . C762

6 Conclusions

C764

References

C765

\section{Introduction}

In both regulated and market-based wholesale electricity systems, the shortterm and long-term predictions of power demand are crucial to operating a secure and reliable system. Electricity demand fluctuates from hour to hour, 
day to day and season to season, based on the aggregate domestic, commercial and industrial loads attached to the network. Forecasting activities performed by the power system operator and by market participants involve estimating expected outcomes as a point forecast, but also establishing the statistical dynamics of the system demand. Periods of high demand create stresses on transmission and distribution network infrastructure, create opportunities for generators and introduce significant financial risks for electricity retailers. Modern deregulated electricity markets exhibit extremely volatile spot prices set by complex market rules involving power station supply offers and the prevailing system demand [1]. In this article, we present a simple diffusion equation (1) to model electricity demand in the New South Wales (NSW) region of the Australian National Electricity Market (NEM). This study fulfils several objectives, as detailed in the following points.

1. We present a parsimonious three-parameter model for describing the stochastic behavior of electricity demand.

2. A calibration approach is developed to fit the model parameters, applying a moment-matching technique.

3. The performance of the model is assessed to represent the statistical dynamics of historical demand data using in-sample and out-of-sample tests.

\section{Electricity demand}

Electricity demand data represents the aggregate instantaneous consumption of electrical power by all loads (or equivalently the aggregate production of electricity by generators). Electricity demand in the NEM is captured by the many electricity meters installed in the electricity network and reported by the market operator AEMO in half-hourly resolution. The demand time series contains several anthropomorphic characteristics, including a diurnal shape, a weekly pattern with distinct demand levels for different day types 
and a seasonal pattern across the year with demand peaking in summer and winter. Electricity demand exhibits a degree of unpredictability, which is driven by random deviations in consumption, which are in turn motivated by weather, economic growth, major sporting events, power distribution network failures and myriad other factors [4, 11, 9]. Diffusion equations (Ito stochastic differential equations) are applied widely through physical and social sciences to model time-continuous quantities exhibiting uncertainty. The electricity demand process appears ideal to model with this technique owing to the high frequency of the data (closely approximating a continuous-time process) and the deviations which are driven by continuous changes in usage by many individual consumption agents. For the current study, data is available from the Australian National Electricity Market which disseminates 5 minute and 30 minute demand data over a 14 year history. The figures in this article illustrate samples of electricity demand.

Extensive research was carried out by other authors using a variety of modeling techniques to predict electricity demand. Neural networks [13], regression methods [6], time series techniques [15, 7], econometric modeling [16] and singular value decomposition methods [8] have all been applied. Other authors conducted modeling of the demand as a stochastic process depending on a latent variable (typically weather) $[18,6]$ or without any exogenous dependencies [17].

\section{Model formulation}

\subsection{Stochastic differential equation}

We model electricity demand with an Ornstein-Ulenbeck equation [12] containing a time dependent drift term. Similar models [2, 9, 12] analysed either 
demand or price processes. Denote aggregate electricity demand as $\mathbf{S}$, defined by a time-continuous stochastic process:

$$
d S(t)=\theta[\mu(t)-S(t)] d t+\sigma d W(t) .
$$

Here $\theta$ is a mean reversion rate, $\mu(t)$ is a time dependent function which provides a target level for the mean reversion and $\sigma$ is the arithmetic volatility. In model (1), the fluctuations arising from weather outcomes are contained in the volatility factor $\sigma$. The model is enhanced to

$$
\mathrm{d} S(\mathrm{t})=\theta[\mu(\mathrm{t}, \mathrm{T})-\mathrm{S}(\mathrm{t})] \mathrm{dt}+\sigma \mathrm{dW}(\mathrm{t}),
$$

with a more complex drift term dependent on both time and temperature $T(t)$. However, the model only has utility if a suitable forecast or governing process for $T$ is available.

\subsection{Characteristics of SDE solution}

The solution of the SDE is established by a standard technique [12] by employing Ito's Lemma and applying an integrating factor.

Lemma 1. The solution to the initial value problem (1) is

$$
S(t) \exp (\theta t)=S(0)+\int_{0}^{t} \theta \mu(\tau) \exp (\theta \tau) d \tau+\int_{0}^{t} \exp (\theta \tau) \sigma d W(\tau)
$$

Lemma 2. The solution to initial value problem (1) has the first moment, variance and skewness, respectively,

$$
\begin{aligned}
\mathrm{E}[\mathrm{S}(\mathrm{t})] & =\mathrm{S}(0) \exp (-\theta \mathrm{t})+\exp (-\theta \mathrm{t}) \int_{0}^{\mathrm{t}} \theta \mu(\tau) \exp (\theta \tau) \mathrm{d} \tau, \\
\operatorname{Var}[\mathrm{S}(\mathrm{t})] & =\frac{\sigma^{2}}{2 \theta}[1-\exp (2-\theta \mathrm{t})], \\
\mathrm{E}\left([\mathrm{S}-\mathrm{E}(\mathrm{S})]^{3}\right) & =3 \exp (-\theta \mathrm{t}) \mathrm{S}(0) \mathrm{E}(\mathrm{S})\left(\frac{\sigma^{2}}{2 \theta}-1\right) .
\end{aligned}
$$


Proof: Taking first moments of (3) and exploiting the fact that the expectation of an Ito integral is zero [12] yields expression (4). Taking the variance of each side of (3),

$$
\begin{aligned}
\exp (2 \theta t) \operatorname{Var}[S(t)] & =\sigma^{2} E\left(\int_{0}^{t} \exp (\theta \tau) d W(\tau)\right)^{2} \\
& =\frac{\sigma^{2}}{2 \theta}[\exp (2 \theta t)-1]
\end{aligned}
$$

yielding the result for the second moment. The third and higher moments follow in a similar way.

Lemma 3. Throughout, define $\overline{\mathrm{S}}(\mathrm{t})=\mathrm{E}[\mathrm{S}(\mathrm{t})]$. It follows that

$$
\mu(t)=\frac{1}{\theta} \frac{d}{d t} \bar{S}(t)+\bar{S}(t) .
$$

Proof: Apply expression (4) to give

$$
\bar{S}(t)=\exp (-\theta t)\left[S(0)+\int_{0}^{t} \theta \mu(\tau) \exp (\theta \tau) d \tau\right] .
$$

Differentiate with respect to $t$ and rearrange to yield the result.

Lemma 4. The autocorrelation of the process governed by (3) with lag $\lambda$ is

$$
\rho[S(t+\lambda), S(t)]=\left(\frac{1-\exp (-2 \theta t)}{1-\exp [-2 \theta(t+\lambda)]}\right)^{\frac{1}{2}} \exp (-\lambda \theta) .
$$


Proof: The proof follows analogously to the case of constant $\mu$,

$$
\begin{aligned}
\rho[S(t+\lambda), S(t)] & =\frac{E[S(t+\lambda) S(t)]-E[S(t+\lambda)] E[S(t)]}{\{\operatorname{Var}[S(t+\lambda)] \operatorname{Var}[S(t)]\}^{1 / 2}} \\
& =\frac{E[S(t+\lambda) S(t)]-\bar{S}(t+\lambda) \bar{S}(t)}{\left\{\frac{\sigma^{2}}{2 \theta}[1-\exp (-2 \theta(t+\lambda)][1-\exp (-2 \theta t)]\}^{1 / 2}\right.} .
\end{aligned}
$$

The expression simplifies to the result by exploiting the fact that expectations of the diffusion terms are zero.

\subsection{Demand regression model}

To perform the calibration of the SDE, a key element in our method involves the mathematical expectation for the demand at each point in time. We apply a regression model to estimate the expected demand and to later generate estimates for variance and other statistics. Our approach applies a common industry technique of segmented regressions. The basic regression model is further enhanced to include temperature. For the basic regression model consistent with SDE (1), we define the model for expected demand D dependent on time $t$ in years, commencing from 1-Jan-2000, and a seasonal characteristic. The seasonality contains an annual and biannual frequency, attributable to both summer and winter events causing elevated demand periods. Define the $8 \times 48$ segmentations:

- Daytype $d \in\{$ Mon, Tues, Wed, Thu, Fri, Sat, Sun, Public Holiday $\}$.

- Period $p \in\{1,2, \ldots, 48\}$, is the halfhourly period of each day.

We let

$$
\begin{aligned}
D= & f\{d, p\}(t) \\
= & a_{0}\{d, p\}+a_{1}\{d, p\} t+a_{2}\{d, p\} \cos (2 \pi t)+a_{3}\{d, p\} \sin (2 \pi t) \\
& +a_{4}\{d, p\} \cos (4 \pi t)+a_{5}\{d, p\} \sin (4 \pi t) .
\end{aligned}
$$


For the extension to accommodate temperature in model (2), the basic regression is enhanced on the basis of a biquadratic relationship for temperature. The quadratic dependence is a well studied behavior and is empirically supported by our case study in Section 5 . Let $T_{\min }$ and $T_{\max }$ represent the daily minimum and maximum temperatures, respectively, at a nominated weather station. We extend model (9) to a temperature-dependent regression by

$$
\begin{aligned}
D= & g\{d, p\}\left(t, T_{\min }, T_{\max }\right) \\
= & a_{0}\{d, p\}+a_{1}\{d, p\} t+a_{2}\{d, p\} \cos (2 \pi t)+a_{3}\{d, p\} \sin (2 \pi t) \\
& +a_{4}\{d, p\} \cos (4 \pi t)+a_{5}\{d, p\} \sin (4 \pi t)++b_{1}\{d, p\} T_{\min }+b_{2}\{d, p\} T_{\max } \\
& +b_{3}\{d, p\} T_{\min } T_{\max }+b_{4}\{d, p\} T_{\text {min }}^{2}+b_{5}\{d, p\} T_{\max }^{2} .
\end{aligned}
$$

\section{Model calibration approach}

\subsection{Literature}

Our objective is to establish suitable parameters in (1) and (2) to best describe the statistical dynamics of demand. Using a direct naïve substitution, one may (falsely) assert that

$$
\mu(t)=\bar{S}(t) .
$$

It is standard practice in industry to denote $\mu(t)$ as the 'mean reversion level' for expression (1) (and variants of that equation). The popular text by Geman [9] which is used extensively by practitioners also developed equation (1) as a mean reverting model for price rather than demand. While $\mu(t)$ is described as the 'trend', by Geman, the text contains no specific calibration for the form of $\mu(t)$. Alcock et al. [2] developed a model of the form

$$
d S_{t}=\alpha\left[S^{*}-\log \left(S_{t}\right)\right] S_{t} d t+S_{t} \sigma d W+S_{t} K d q_{t},
$$


and refered to $\mathrm{S}^{*}$ as the mean reversion level. Through the development and calibration of the model, they arrived at an intermediate expression containing the equivalent of our equation (7). Goard and Hansen [10] also performed calibration of a similar SDE using a generalized method of moments. Blanco and Soronow [3] presented a version of (1) and described the mechanics of mean reversion driven by the drift. They used a constant $S^{*}$ in place of our term $\mu(t)$ and claimed that $S^{*}$ is the long-run equilibrium. The authors provided explicit calibration steps which only remain valid for constant $S^{*}$. However, for the model with time dependent drift it becomes quickly apparent that if the naïve substitution (11) is made, then the first moment of the solution to (1) suffers a time lag to the desired result $\mathbf{S}(\mathbf{t})$. The inaccuracy does not arise for a time independent mean-reversion level.

\subsection{Methodology for calibration of the SDE}

By inspection, the solution (3) is seen to exhibit a Gaussian distribution. Consequently, the controls availed to the calibration process (selection of $\mu$, $\theta$ and $\sigma$ ) yield a solution of limited characteristics and the parameter fitting becomes a process of establishing the best fit according to certain objectives. Although we have three parameters, we are unable to fit the first three moments as the solution is governed by a normal distribution (with only two degrees of freedom).

Our primary condition is governed by matching the first moment. Suppose that the historical mean behaviour or forecast for expected demand is given by $\bar{S}(t)$. Then Lemma 3 yields the first calibration equation (7).

The secondary objective is to match the second moment of the solution. Illustrations of the electricity demand process demonstrate that the demand at any given time can assume a large excursion away from the mean shape. Expression (5) conveys the variance of the solution (3) which clearly approaches the constant $\sigma^{2} / 2 \theta$ as time increases. 
The comparison of historical demand against the fitted demand enables us to estimate the variance of $\mathbf{S}(t)$ at each future point in time on the basis of the residuals arising from the regression. In reality, the variance might have a time dependent structure which is not accommodated within our proposed model containing constant values of $\theta$ and $\sigma$, but this is beyond the capabilities of the proposed parsimonious model.

Let $V(t)$ be the projected variance of $S(t)$ at each point in time over a future horizon $[0, T]$. The optimal fit to match the second moment in a least squares sense is

$$
\min _{\sigma, \theta}\left[\int_{0}^{T}\left(V(t)-\frac{\sigma^{2}}{2 \theta}[1-\exp (-2 \theta t)]\right)^{2} d t\right] .
$$

If we assume that $\mathrm{T}$ is large (that is, the demand model is designed to project over a long future horizon), and make the a priori assumption that our calibration yields $\theta>0$, then $\exp (-2 \theta t)$ is treated as transient. The minimization (12) is then achieved by

$$
\frac{\sigma^{2}}{2 \theta}=\frac{1}{T} \int_{0}^{T} V_{\tau} d \tau
$$

which is interpreted as the time-averaged variance.

Expressions (7) and (13) leave one degree of freedom remaining in specifying the model parameters. However, expression (6) conveys there is no benefit in matching higher moments since the Gaussian nature of the distribution is predetermined. When we inspect the nature of the trajectories of solutions to (3) generated by parameters from the family satisfying (7) and (13), it becomes apparent that the solutions differ by their 'clustering' behaviour. Matching the first two moments represents insufficient conditions to yield a unique solution. Candidate solutions arise which yield small or large mean reversion strength $\theta$. The respective behaviour of the trajectories is qualitatively described by slowly varying or rapidly varying envelopes of daily demand patterns and so an additional statistic of the solution is required to uniquely determine the SDE parameters. 
Formally, we approach the parameter fitting by matching the autocorrelation of observed data with the characteristics of the solution (3). For $\lambda>0$, Lemma 4 exposes the asymptotic behaviour:

$$
\rho(S(t+\lambda), S(t)) \rightarrow \exp (-\lambda \theta) .
$$

A common technique in statistical data fitting involves detrending data before fitting statistics [5]. However, the approach here matches autocorrelations of the solution $\mathrm{S}(\mathrm{t})$ directly with observed historical demand.

\section{Case study: NSW region of the NEM}

\subsection{Data sources}

The calibration was conducted using historical halfhourly demand from the NSW region of the NEM. The in-sample period for the regression covered 1-Jan-2000 to 30-Jun-2011. The out-of-sample testing was performed over 1-Jul-2011 to 30-Jun-2012. The weather station for temperature dependence was Sydney Airport. Measures of the goodness of fit of the model were performed by visual inspection of the time series, by comparisons of the duration curve and through the residual error.

\subsection{Regression fit}

The regression models (9) and (10) were implemented on the in-sample data. Figures 1 and 2 show the nature of the data exhibiting daily and weekly patterns. We illustrate load shapes during a typical summer and winter week to convey the way that the shape and quantum alter between seasons. The regression fit yields the quantity $\bar{S}(t)$. 


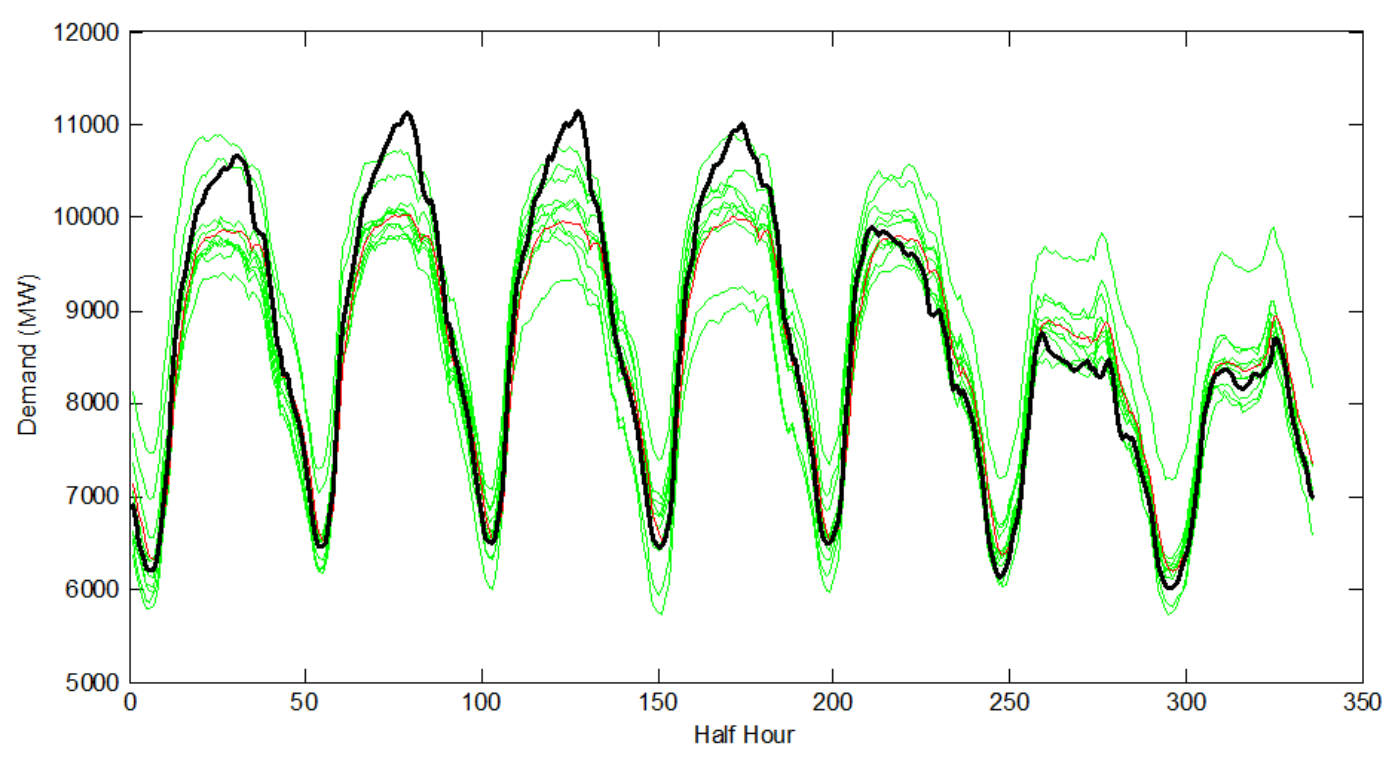

Figure 1: Simulated trajectories (green) from expression (1) for week starting 23-Mar-2009, regression fit (red) and historical (black).

\subsection{Calibration of the SDE}

The SDE calibration proceeds by solving the system of equations (7), (13) and (14). The lag $\lambda$ was chosen as one day on the basis of the standard daily cycle of demand patterns, that is $\lambda=0.0027$ years.

The historical autocorrelation of demand data is calculated as 0.8917. Solving (14) yields $\theta=45.006$ year $^{-1}$. Using alternative units, $\theta \approx 1 / 8$ day $^{-1}$, or in other words the half-life of a return to mean is $\log (0.5) / \theta=5.6$ days, consistent with the typical half-life of weather patterns [14]. The expression (7) is now solved by direct substitution of $\theta$ and the regression outcome $\bar{S}(t)$. We use a one-sided finite differencing to estimate $d \bar{S}(t) / d t$. Finally, solving (13) yields $\sigma=4,605.9$.

The $R^{2}$ from the regression fit was 0.86 . We interpret this in the standard 


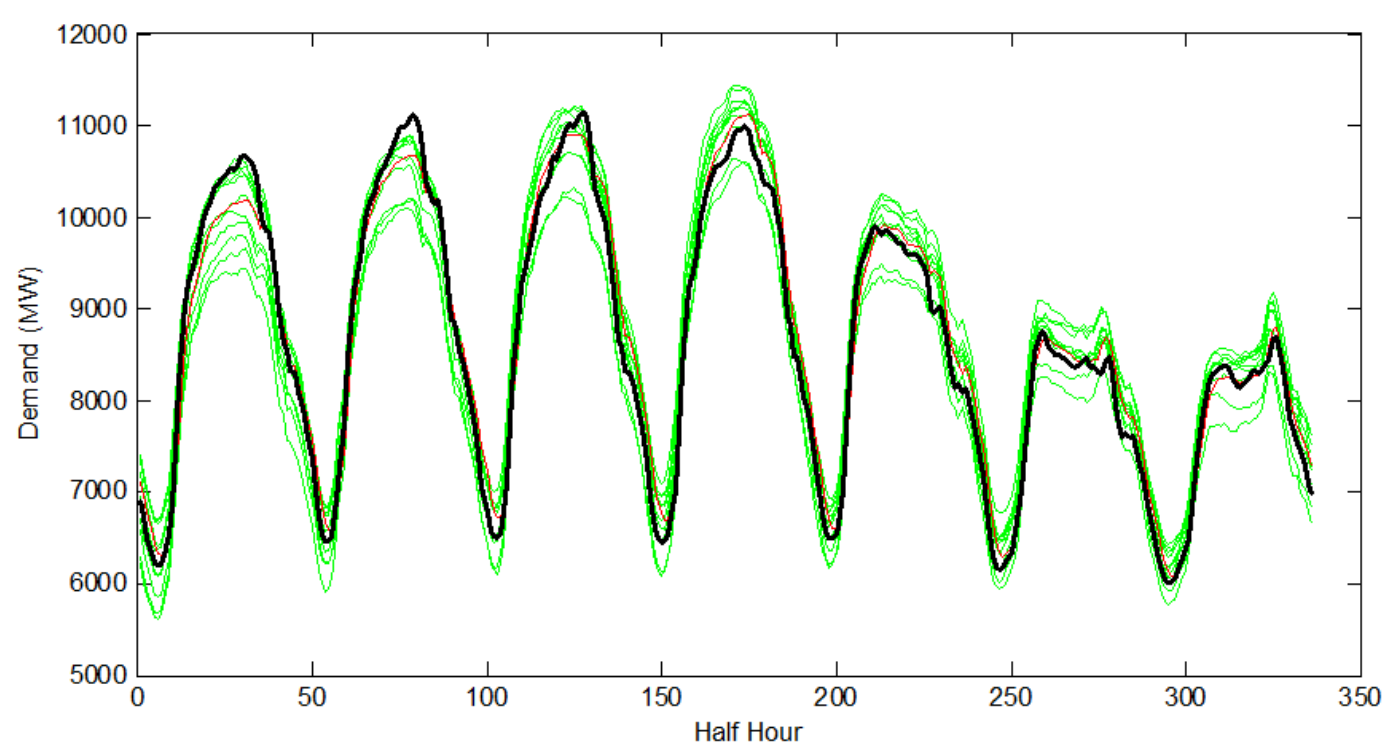

Figure 2: Simulated trajectories (green) from expression (2) for week starting 23-Mar-2009, regression fit (red) and historical (black).

way that the daytype, time of day, season and point in the growth cycle was able to explain $86 \%$ of the variability in demand. The standard error (standard deviation of regression residuals) was $485 \mathrm{MW}$, compared with typical demands around $9000 \mathrm{MW}$.

When the model was enhanced with the temperature dependence, an improved regression was achieved with $\mathrm{R}^{2}=0.91$ and standard error diminished to $411 \mathrm{MW}$.

\subsection{Results}

The figures here represent results of the simulations arising from expressions (1) and (2) on the basis of calibration conducted by the methodology described in Section 5.3. 


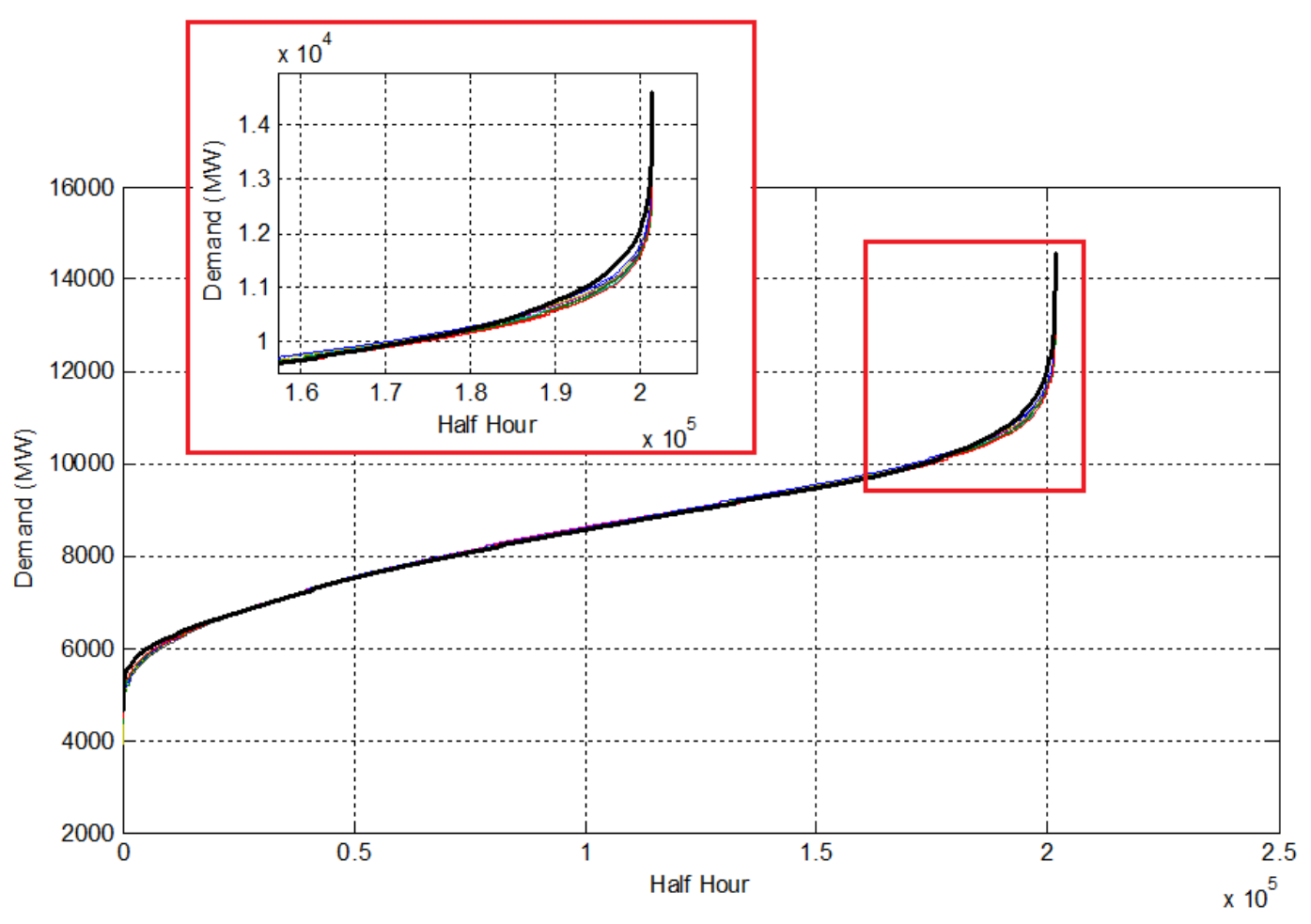

Figure 3: Duration curve for solutions from unconditional model over insample period for historical data (black) and simulated trajectories. Inset: zoom into top end of curve.

\subsubsection{In sample simulations}

Firstly, for a typical in-sample week we present the actual NSw demand and the regression-fitted demand for the same period using the unconditional model and the temperature dependent model. The plots also contain ten simulations of the SDEs (1) and (2) to illustrate the distribution of demand trajectories which may feasibly occur during the given time period.

The duration curve is a standard visualization used in the energy industry for 


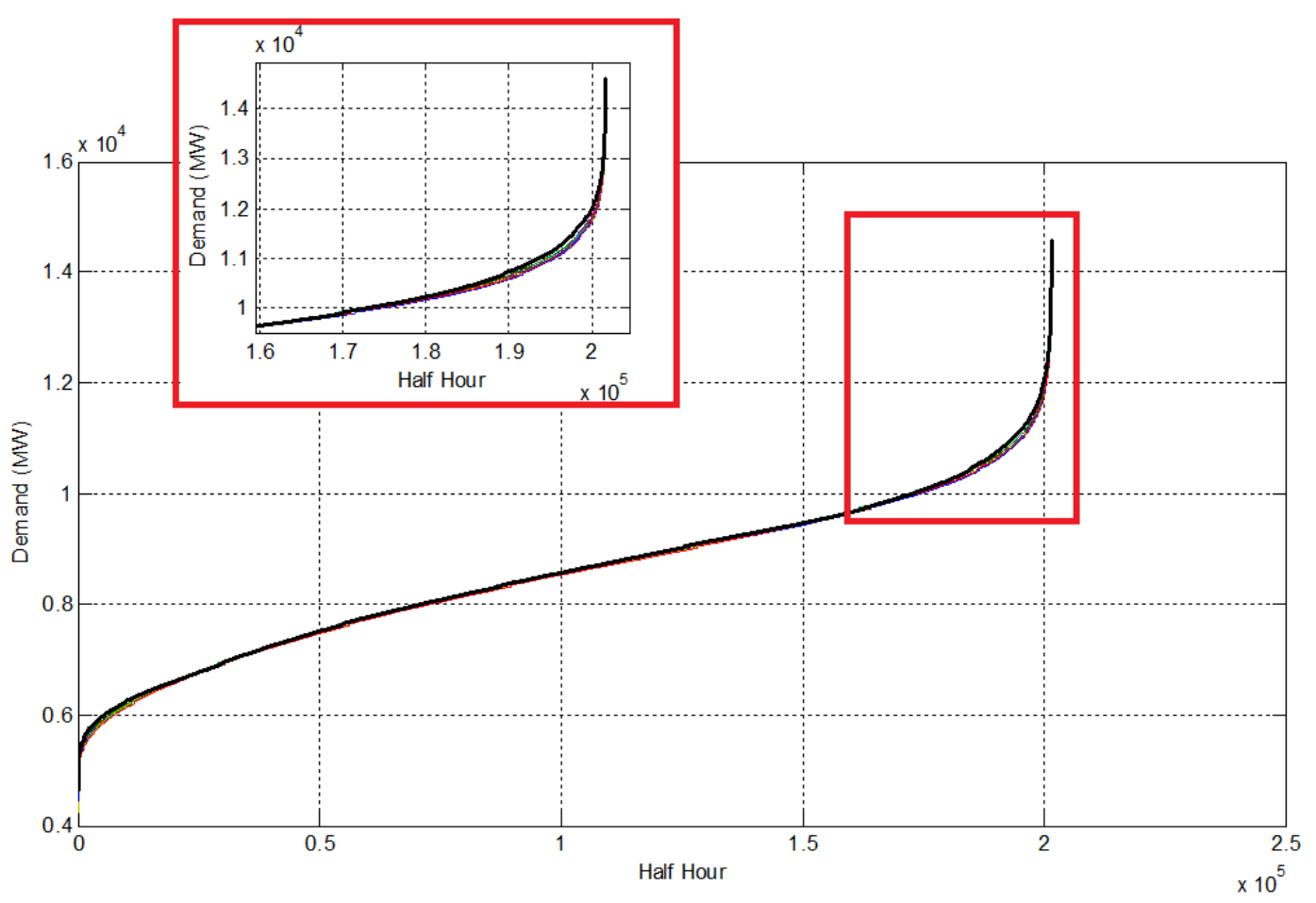

Figure 4: Duration curve for solutions from temperature dependent model over in-sample period for historical data (black) and simulated trajectories. Inset: zoom into top end of curve.

presenting the cumulative distribution of outcomes [1] and simply represents a plot of the sorted data. Figures 3 and 4 show the actual duration curve and the results of ten simulations over the full historical calibration period. Importance is assigned to the characteristics of the curve in the upper percentiles, because this is the region that eventuates in most financial risk. It is clearly seen that the temperature dependent model outperforms the unconditional model. 


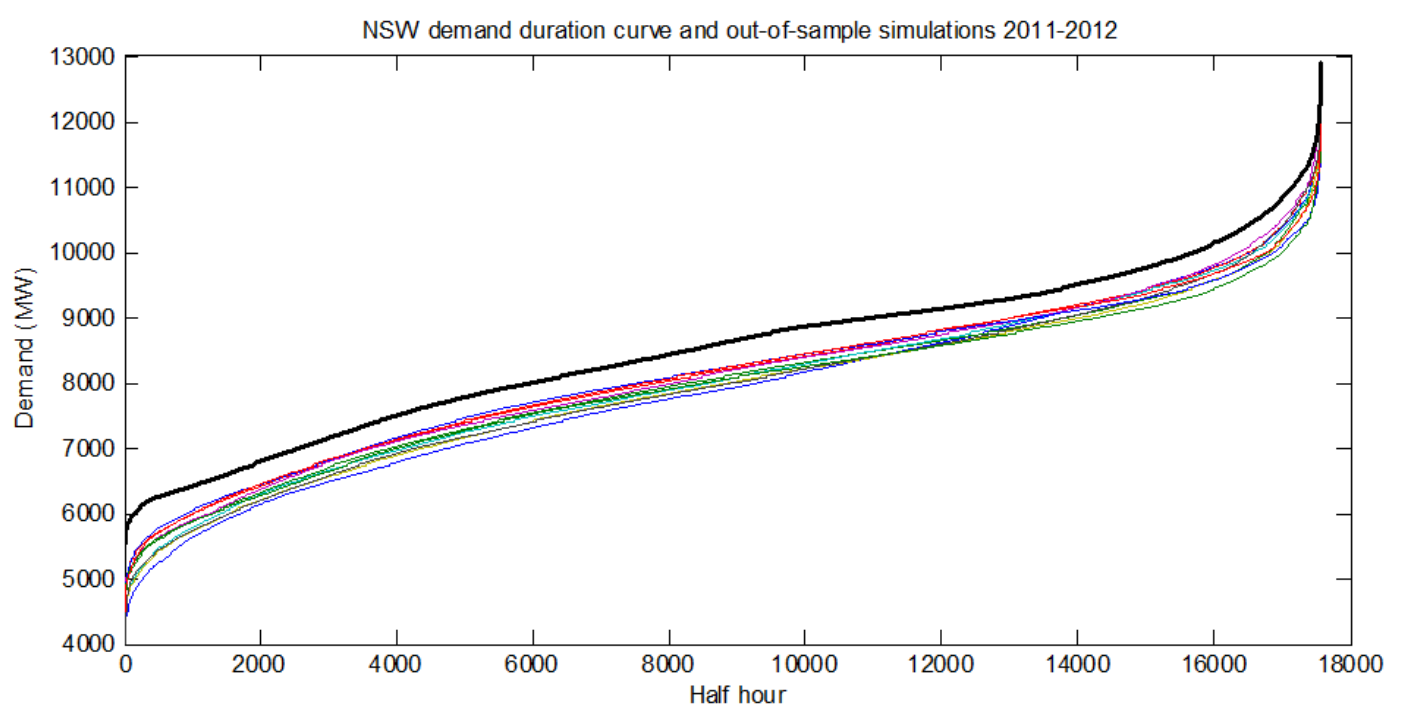

Figure 5: Duration curve for solutions from temperature dependent model over out-of-sample period for historical data (black) and simulated trajectories.

\subsubsection{Out of sample simulations}

Figure 5 shows the out-of-sample simulations compared with actual NSW demand outcomes over Jul-2011 to Jun-2012. The results illustrate that since 2011 there has been a step-change downward in electricity demand. It is unlikely to be attributable to solar photovolatic generation because the drop is almost uniform over all times (including midnight when solar is clearly not being generated). There were several large industrial closures in NSw, for example, the Hydro Kurri Kurri smelter, which may explain the result.

\subsubsection{Earth Hour}

The Earth Hour event is an awareness campaign which aims to make the consumer public conscious of energy useage. Its key event is a nominated hour 


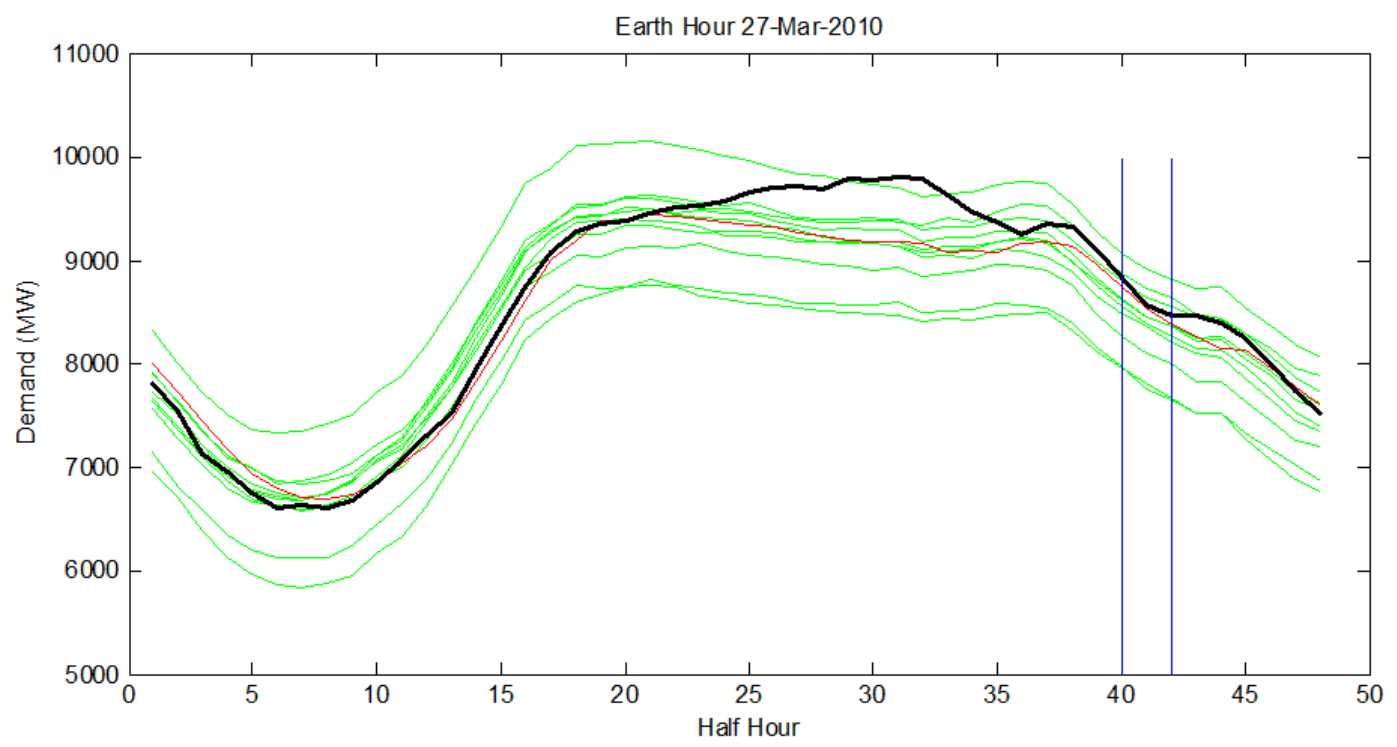

Figure 6: In sample models of the 2010 Earth Hour actual demand, regressed demand and simulations, conditioned upon known Sydney temperatures. The Earth Hour period is between the vertical blue lines.

each year in which consumers are encouraged to reduce electricity demand. The event is held at a nominated local time. Sydney is typically on daylight savings time when Earth Hour event takes place. However, the NEM operates on Australian Eastern Standard Time.

We are able to use the regression model to establish the expected demand during Earth Hour, and compare with the actual demand to establish the net curtailment effects. The simulations of the SDE provide confidence bands in our forecast to enable us to establish the significance of any observed reduction in consumption.

Figure 6 presents result for Earth Hour 2010, which occurred on 27 March 2010 from 8:30 to $9: 30 \mathrm{pm}$ local time (NEM periods 40 to 41). Most interestingly, the demand exhibits a slight rise above trend after Earth Hour, which may 
be explained by consumers simply deferring energy consumption rather than reducing consumption.

The results illustrate that the level of curtailment for Earth Hour is almost indistinguishable, and well within the level of noise regularly experienced.

\section{Conclusions}

The parsimonious models presented in (1) and (2) perform reasonably to describe the deterministic and stochastic components of NSW regional electricity demand. The introduction of temperature as a predictor variable in the regression improved the model substantially, with residuals around $411 \mathrm{MW}$ from the best regression.

The very simple model also contains known deficiencies. For example, the volatility and mean reversion rates may themselves be time dependent. The growth pattern was formulated as a linear relationship. However, for practical purposes, the simplicity of this model provides a useful working model as it enables explicit solution, quick and transparent calibration and rapid simulation.

The calibration approach developed in this article leads to a very transparent method for parameter estimation. It leads to uncoupled equations, in contrast to techniques such as maximum likelihood which yield large systems of equations requiring numerical solution.

An important exposition shows that $\mu(t)$, which is referred to throughout industry and in many texts as the mean reversion level, is not, in fact, the level to which the process targets. Instead its calculation by (7) is vastly different from $\bar{S}(t)$.

The model detected that the growth trend apparent through 2000-2011 does not apply to 2012 and there is a reduction in electricity demand across all of the stratifications by day type and time-of-day. 
To the accuracy of the regression model, and within the confidence bands arising from the diffusion process, the effects of demand reduction arising during Earth Hour events were of negligible impact.

\section{References}

[1] An Introduction to Australia's National Electricity Market, July 2010, Australian Energy Market Operator Limited, Accessed from http://www . aemo.com.au/corporate/0000-0262.pdf March 2011. C749, C761

[2] J. Alcock, J. Goard and T. Vassallo, Calibrating Mean Reverting Jump Diffusion Models: An Application to the NSW Electricity Market, in T. Marchant Ed. Proceedings of the 2007 Mathematics and Statistics in Industry Study Group, MISG, Wollongong, 57-81, 2007.

http://www. uow.edu.au/content/groups/public/@web/@inf/ Omath/documents/doc/uow040974.pdf C750, C754

[3] C. Blanco and D. Soronow, Mean Reverting Processes-Energy Price Processes Used for Derivatives Pricing and Risk Management, Commodities Now, June, 68-72, 2001.

http://www.fea.com/resources/a_mean_reverting_processes.pdf $\mathrm{C} 755$

[4] S. R. Brubacher and G. Tunnicliffe Wilson, Interpolating Time Series with Application to the Estimation of Holiday Effects on Electricity Demand, J. Royal Statistical Society Series C, 25:107-116, 1976. http://www. jstor.org/stable/2346678 C750

[5] N. R. Draper, and H. Smith, Applied Regression Analysis, Wiley Series in Probability and Statistics, 1998. C757 
[6] R. F. Engle, C. Mustafa, J. Rice, Modelling peak electricity demand, Journal of Forecasting, 11:241-251, 1992. doi:10.1002/for.3980110306 C750

[7] E. Erdogdu, Electricity demand analysis using cointegration and ARIMA modelling: A case study of Turkey, Energy Policy, 35:1129-1146, 2007. doi:10.1016/j.enpol.2006.02.013 C750

[8] F. Li, Singular value decomposition expansion for electrical demand analysis, IMA Journal of Mathematics Applied in Business and Industry, 11:37-48, 2000. doi:10.1093/imaman/11.1.37 C750

[9] H. Geman, Commodities and Commodity Derivatives, Wiley, Chichester, 2005. C750, C754

[10] J. M. Gourd and N. Hansen, Comparison of the performance of a time-dependent short-interest rate model with time-dependent models, Applied Mathematical Finance, 11:147-164, 2004. doi:10.1080/13504860410001686034 C755

[11] S. Mirasgedis, Y. Sarafidis, E. Georgopoulou, D. P. Lalas, M. Moschovits, F. Karagiannis and D. Papakonstantinou, Models for mid-term electricity demand forecasting incorporating weather influences, Energy, 31:208-227, 2006. doi:10.1016/j.energy.2005.02.016 C750

[12] B. Oksendal, Stochastic Differential Equations: An Introduction with Applications, 4th ed., Springer-Verlag, New York, NY, 1995. C750, C751, C752

[13] B. R. Szkuta, L. A. Sanabria, T. S. Dillon, Electricity price short-term forecasting using artificial networks, IEEE Trans Power Systems, 14:851-857, 1999. doi:10.1109/59.780895 C750

[14] B. Petschel, Mean Reversion Models for Weather Derivatives, PhD Thesis, University of Queensland, 2005 C758 
[15] J. W. Taylor, Short-term electricity demand forecasting using double seasonal exponential smoothing, Journal Operational Research Society, 54:799-805, 2003. doi:10.1057/palgrave.jors.2601589 C750

[16] S. C. Tripathy, Demand Forecasting in a Power System, Energy Convers. Mgmt. 38:1475-1481, 1997. doi:10.1016/S0196-8904(96)00101-X C750

[17] R. Weron and A. Misiorek, Modeling and Forecasting Electricity Loads: A Comparison, in Proceedings of The European Electricity Market EEM-04 Lodz, Poland, 135-142, 2004. C750

[18] C. J. Ziser, Z. Y. Dong, T. Saha, Investigation of Weather Dependency and Load Diversity on Queensland Electricity Demand, in M.

Negnevitsky Ed. Proceedings of Australasian Universities Power

Engineering Conference 2005, AUPEC, Hobart, 2:457-462, 2005. C750

\section{Author addresses}

1. Elliot Tonkes, Energy Edge Pty, Ltd, PO Box 10755, Brisbane 4000, Australia.

mailto: etonkes@energyedge.com.au

2. Phil Broadbridge, School of Engineering and Mathematical Sciences, La Trobe University, Melbourne Victoria 3086, Australia. 